\title{
Téoros
}

Revue de recherche en tourisme

\section{Un lieu, des gens, la vie d'une collectivité dans les}

Hautes-Laurentides

\section{Une étude de cas en matière de tourisme communautaire}

\section{Marie-Andrée Delisle}

Volume 26, numéro 3, automne 2007

Tourisme et solidarité

URI : https://id.erudit.org/iderudit/1071006ar

DOI : https://doi.org/10.7202/1071006ar

Aller au sommaire du numéro

Éditeur(s)

Université du Québec à Montréal

ISSN

0712-8657 (imprimé)

1923-2705 (numérique)

Découvrir la revue

Citer cet article

Delisle, M.-A. (2007). Un lieu, des gens, la vie d'une collectivité dans les

Hautes-Laurentides : une étude de cas en matière de tourisme communautaire.

Téoros, 26(3), 46-50. https://doi.org/10.7202/1071006ar 


\section{Un lieu, des gens, la vie d'une collectivité dans les Hautes-Laurentides Une étude de cas en matière de tourisme communautaire}

\section{Marie-Andrée Delisle}

Que savons-nous du tourisme communautaire? Souvent associé à un tourisme vécu dans l'axe Nord-Sud, comme en Afrique ou en Amérique centrale, nous ne pensons pas qu'il puisse se vivre et s'articuler au Nord. Dans les faits, le cas que nous illustrons ici démontre, à partir d'une formule d'hébergement particulière, que les applications se ressemblent puisqu'elles nécessitent la collaboration et la participation de la communauté qui s'y engage.

Plus précisément, le concept de tourisme communautaire

accorde la plus grande place au processus de prise de décision des résidants d'une communauté visitée, à une forme de gouvernance participative de la population dans le choix des visiteurs qu'elle souhaite recevoir et dans les modalités de leur intégration dans la communauté qu'elle privilégie (visites dans les villages, hébergement ou restauration chez l'habitant...). Le tourisme communautaire a pour objectifs de générer des revenus, de créer des emplois, de réduire la pauvreté et de causer un minimum d'impact sur la culture et l'environnement local (Delisle et Jolin, 2007 : 51).

Cela sous-entend une volonté de solidarité entre les villageois, la formule s'appliquant principalement dans les régions rurales. Une communauté peut donc bâtir un projet touristique à sa mesure, tisser des liens d'entraide, imaginer des solutions et se doter d'une vision de développement viable. Les villages d'accueil, notamment celui des Hautes-Laurentides, en constituent une illustration probante.

\section{Qu'est-ce qu'un village d'accueil ?}

Les villages d'accueil ne sont pas nouveaux au Québec. Existant depuis les années 1970 , ils « offrent, moyennant un prix forfaitaire, des activités d'accueil et d'animation de groupe, de l'hébergement, le petit-déjeuner et le repas du midi ou du soir dans des familles qui reçoivent un maximum de 6 personnes " ${ }^{1}$. La formule a grandement plu aux voyagistes offrant des circuits au Québec à partir de la France: on proposait un lien direct avec "du vrai monde " et on faisait une bonne affaire, la nuitée, les repas et l'animation revenant à des coûts minimes. Plus récemment, soit en 2002, la Corporation de l'industrie touristique du Québec (CITQ) ${ }^{2}$ a commencé à classifier les villages d'accueil au même titre que tous les autres types d'hébergement touristique au Québec ${ }^{3}$. Ainsi, en plus de devoir se conformer à la définition de base, ce type d'hébergement, offert à des groupes, doit respecter certains critères d'accueil; les villages peuvent donc être classifiés de une à quatre étoiles, selon les services offerts. Bon an mal an, les villages ont généré près de 1,5 million de dollars en 2005, procurant un revenu d'appoint à près de 337 familles québécoises (DBSF, 2007 : 4) réparties dans une douzaine de villages.

\section{Un village d'accueil "nouvelle génération " dans les Hautes-Laurentides}

Né de l'idée et de la détermination d'un ancien maire de Kiamika à faire venir et à retenir des visiteurs dans sa région pour leur faire partager une expérience " de terrain », le Village d'accueil des Hautes-Laurentides entend proposer une formule "nouvelle génération" de ce type d'hébergement. Fonctionnant en tant qu'entreprise d'économie sociale, l'organisme a vu le jour grâce à la volonté et à la collaboration des

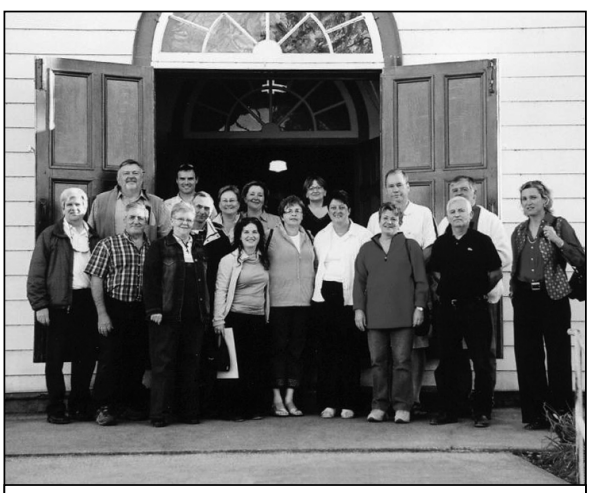

Groupe de travail devant l'église Kiamika, entouré de quelques familles d'accueil. Photo: Marie-Andrée Delisle

municipalités de Kiamika, de Lac-du-Cerf, de Notre-Dame-de-Pontmain et de SaintAimé-du-Lac-des-Îles, situées à proximité de Mont-Laurier.

Grâce à un livre publié à l'occasion des fêtes du centenaire de la communauté (Lacasse et al., 1998), la population de Kiamika a pu revisiter son cheminement, raconté au fil des 700 pages qui ont mis en relief son histoire, son économie forestière et agricole, son environnement, sa vie politique, son développement, ses loisirs et son essence touristique. Une même démarche a été réalisée par Notre-Dame-de-Pontmain, sans toutefois faire l'objet d'un livre.

Il va sans dire qu'un tel projet ne peut s'accomplir sans qu'un sentiment de solidarité se crée entre les différents groupes d'intérêt et incite au ralliement de nombreux acteurs intra-régionaux : en ce sens, le niveau politique et la société civile doivent pouvoir regarder dans la même direction pour parvenir à des résultats positifs. Or, les réels partenariats doivent contenir des étapes d'apprentissage dans l'établissement des relations, ce qui nous mène au sujet de la concertation et du consensus. 


\section{De la concertation au consensus}

Comme la vision de développement de ce nouveau produit misait sur un consensus auprès des différentes communautés, l'idée a d'abord été proposée au Centre local de développement (CLD) de la Municipalité régionale de comté (MRC) d'Antoine-Labelle afin de vérifier non seulement s'il y avait de l'intérêt, mais si l'on pouvait trouver un porteur de ballon pour faire cheminer cette idée. La municipalité de Kiamika, instigatrice du projet, s'est donc mise en frais d'organiser des rencontres avec des familles de la place pour leur exposer le projet. Puis l'engouement a fait son chemin vers les municipalités environnantes dont le maire a également démontré son intérêt à recevoir des visiteurs ${ }^{4}$. D'autres rencontres avec les leaders naturels des quatre municipalités ont mené à la constitution d'un organisme qui est présentement administré par un conseil d'administration composé de quatre maires, d'un représentant des familles et d'un représentant du CLD de la MRC d'Antoine-Labelle qui assurent la coordination et le suivi des opérations. Un plan d'affaires a été élaboré et entériné, puis s'en est suivie une série de consultations en vue d'intégrer une gouvernance participative au projet. Les maires des quatre municipalités se sont assurés, lors de consultations, d'obtenir l'assentiment de leur population qui a affirmé sa volonté de travailler à un projet commun et d'élaborer davantage sur ce type d'hébergement.

Les municipalités ont créé un organisme sans but lucratif sous le nom de Village d'accueil des Hautes-Laurentides, doté d'un conseil d'administration les représentant et pouvant leur dresser un tableau mensuel des étapes franchies et à venir afin qu'elles suivent de près les progrès. Parallèlement, ce projet venait temporiser la préoccupation des villageois concernant la problématique de la foresterie et des fermetures d'usines, grandes sources d'emplois dans la région. Les récents coups durs amenés par ces fermetures ont aussi mobilisé les communautés à réfléchir sur des manières de s'entraider et de collaborer à la recherche de solutions. Ne serait-ce que de maintenir en vie le «dépanneur » du coin ou le seul restaurant du village, le projet de village d'accueil représente une possibilité alternative de survie des services à la population vieillissante des municipalités.
La recherche de familles hôtes a suivi et des rencontres individuelles ont été l'occasion de confirmer non seulement l'intérêt des personnes, mais également leur vision du projet dans son ensemble. À la suite des consultations, et dans la perspective de générer un projet d'économie sociale durable, l'idée de retenir plus longuement les visiteurs a fait son chemin et le CLD a recruté 65 familles hôtes qui ont désiré aller au-delà de la formule initiale en ajoutant des activités et en actualisant ainsi le concept de village d'accueil.

Après avoir analysé les tendances du marché et l'évolution des intérêts des clientèles, il a été convenu qu'une formule plus intime serait proposée à des petits groupes et ne s'adresserait pas nécessairement aux voyageurs en autocar, dans le but de conserver une certaine intimité dans la rencontre avec les visiteurs et le partage que l'on entend leur faire vivre.

Avec les responsables de la coordination du projet, nous avons travaillé, à titre de consultante en développement touristique, à l'élaboration d'un inventaire des aspects naturels, humains, patrimoniaux et géographiques des municipalités qui a permis de jeter les bases d'une programmation dynamique donnant lieu à la participation des villageois, des artisans et des experts de la région. En répartissant l'expérience sur un minimum de deux nuits, nous pouvions ainsi y insérer une pleine journée d'activités participatives et d'apprentissage sur le milieu de vie ambiant, faisant en sorte que l'expérience humaine soit non seulement mémorable, mais significative, tant pour le visiteur que le visité.

On ne parle pas tant ici de théâtralisation comme l'entendent Joseph B. Pine et James H. Gilmore (1999) dans leur livre sur l'économie de l'expérience, car l'expérience en soi n'est pas considérée comme du «travail » par les villageois. C'est plutôt une question d'immersion et de participation active qui vise à créer des souvenirs de part et d'autre. Nous retiendrons cependant de ces auteurs leur perspective de l'expérience, dans le sens où elle engage l'individu d'une manière toute personnelle (Idem : 12). Plutôt que d'élaborer une mise en scène, on misera sur le contenu de la programmation comme script dont chaque partie doit être harmonisée.

\section{L'évolution du projet}

L'idée de réinventer la formule du village d'accueil par le biais d'activités a donné un élan additionnel à ce qui est devenu un projet communautaire majeur. Les familles ont étroitement participé aux formations individuelles qui leur ont été prodiguées. En outre, les responsables du projet ont reçu une formation portant sur la création d'activités dites «privilège », en vue d'offrir une expérience unique aux groupes. Leur rôle était aussi de s'assurer que chaque municipalité soit bien représentée dans l'élaboration de ces activités afin d'éviter le favoritisme. Ainsi, le travail de vision et la transparence du déroulement du projet continuent d'être des atouts majeurs de motivation et de collaboration.

D'ailleurs, les objectifs poursuivis par le Village d'accueil des Hautes-Laurentides concordent directement avec ceux des municipalités depuis le début du projet, soit de:

- Prioriser l'utilisation de l'ensemble des ressources du territoire.

- Susciter l'intérêt de la collectivité pour consolider ou démarrer des petites entreprises.

- Intéresser les municipalités dans le développement de nouvelles attractions.

- Travailler en étroite collaboration avec l'Association touristique régionale des Laurentides en tant que partenaire de promotion.

- La création et la programmation de ces activités reposent sur les objectifs suivants :

- Éviter le déjà vu : la quête d'authenticité s'inscrit comme une tendance lourde dans la demande touristique, car les visiteurs recherchent un contact réel, hors des sentiers battus; à ce volet, la recherche de spécificité devient le fil conducteur du projet.

- Viser une expérience humaine dont le contenu pourra mettre en valeur le lieu et les gens, d'où l'idée d'associer la rencontre de familles et la participation à des activités propres au milieu.

- Associer culture et nature afin de faire découvrir un cachet authentique et de faire vivre des moments mémorables dans un encadrement de qualité.

- Axer les activités sur la démonstration de connaissances et d'expertises locales: foresterie, trappe, productions locales, cuisine régionale, cours d'eau, etc. 
- Mettre en valeur la couleur de chaque famille : qui un accent, qui un talent, qui un spécialiste, qui un conteur, qui une mémoire, etc.

La programmation s'est ainsi organisée autour d'une carte culturelle des lieux et autour d'une démarche visant à offrir des activités distinctives, pour les offres estivales et hivernales. Quant à l'authenticité recherchée, elle se trouve principalement dans la véracité des gens et la rencontre du "vrai monde", pour emprunter une expression populaire, tant pour refléter la réalité d'aujourd'hui que pour faire appel au passé et au futur.

\section{L'engagement des familles}

Au fil des visites individuelles des responsables du projet dans chaque maison et des rencontres d'information et de formation, les familles et les responsables des municipalités ont participé à la définition du projet et des objectifs à poursuivre. À ce titre, les familles visent davantage à se reconnaître dans un projet qui leur ressemble et à perpétuer un sentiment d'appartenance à la communauté, les retombées financières venant en second lieu.

En discutant des critères de classification exigés par la Corporation de l'industrie touristique du Québec, les familles ont opté pour l'obtention d'un quatre étoiles et les responsables du projet ont été chargés d'organiser des formations ponctuelles à cet égard, en vue d'assurer un accueil chaleureux constant et de maintenir un service de qualité élevé. Le Village d'accueil des Hautes-Laurentides se veut donc un produit distinctif et il vise à éviter le bas de gamme, gardant en tête l'idée d'un tourisme équitable pour les hôtes comme pour les visiteurs. Par tourisme équitable, on entend ici et de façon globale le fait de « payer un juste prix au fournisseur local pour assurer sa pérennité » (Delisle et Jolin, 2007 : 48).

Dans le but de se préparer à cette expérience, les familles ont été invitées à vivre un séjour d'hébergement en famille dans un autre village d'accueil, ce qui a grandement contribué à les rassurer et à les motiver encore davantage. Un groupe test a également évalué le produit ; composé de professionnels de l'industrie, ce groupe a pu faire bénéficier aux familles de nombreux commentaires et suggestions afin de bonifier l'expérience.

\section{Les opportunités}

En plus d'améliorer leur offre touristique, et sachant que la formule d'hébergement en famille plaît beaucoup aux Européens francophones, le projet a enthousiasmé les municipalités pour plusieurs raisons: le bassin potentiel de clientèles est intéressant, la création d'emplois pour les artistes, les artisans, les coordonnateurs, les musiciens, les animateurs et les petites entreprises locales est réelle, les services touristiques actuels peuvent en bénéficier, la possibilité d'augmenter les revenus des familles et de diversifier l'économie des municipalités réceptives est valable.

Concrètement des partenariats ont été élaborés avec cinq entreprises de la région, une banque d'artistes et d'artisans talentueux a été développée et le Fonds d'économie sociale a favorablement accueilli le projet et versé, en 2006 et en 2007, un montant substantiel qui a permis l'embauche de la coordonnatrice. Le CLD a également investi pour défrayer le salaire et les frais de déplacement des deux responsables du projet.

De plus, le projet amène une synergie et une ambiance de fête au sein des communautés souvent moins actives. En positionnant la région grâce à cette formule

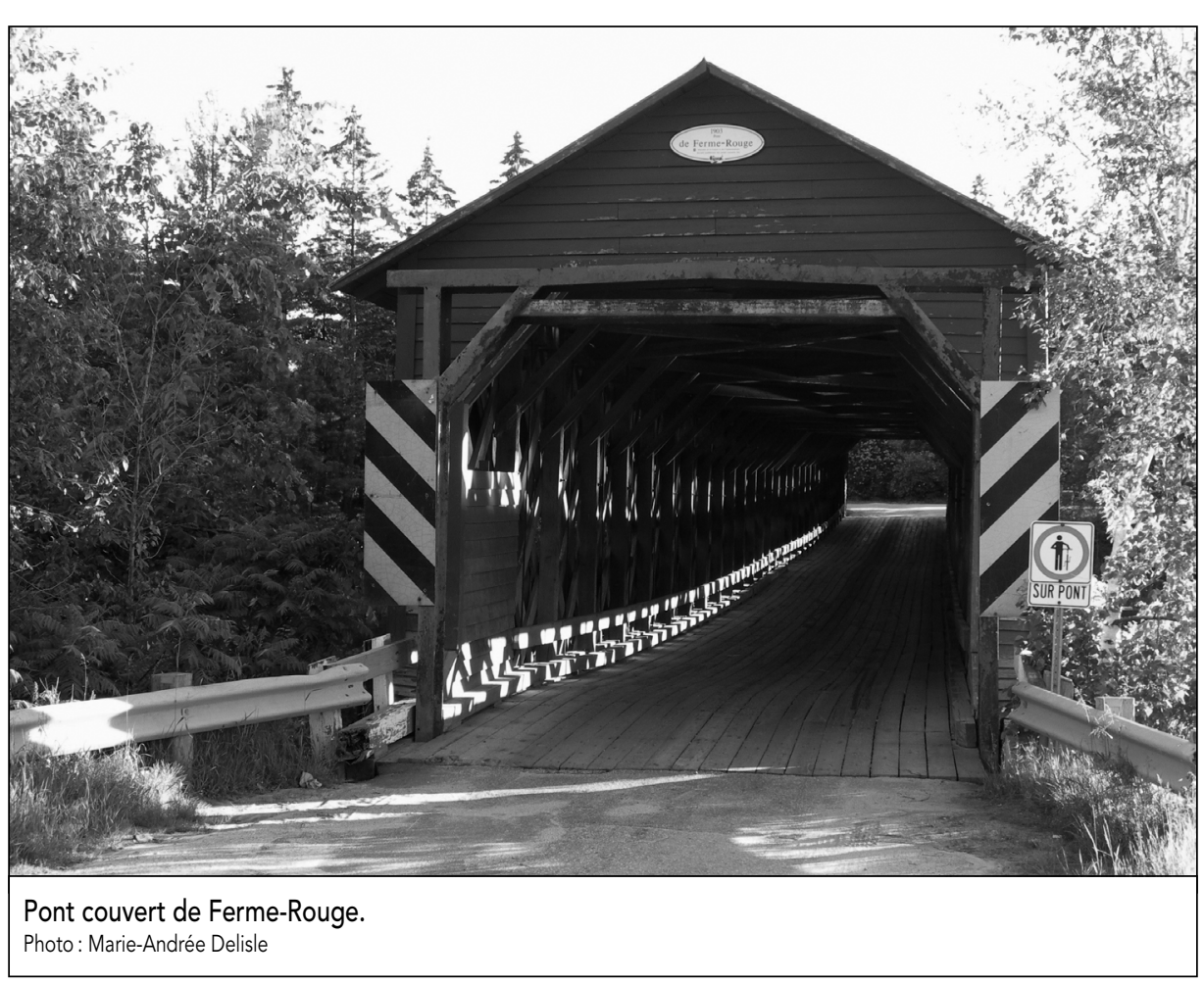

renouvelée, le village d'accueil assure une notoriété aux Hautes-Laurentides, notoriété qui vient enrichir la région touristique des Laurentides dans son ensemble. Par ailleurs, les responsables du projet travaillent présentement à élaborer des collaborations avec les régions limitrophes de l'Outaouais et de l'Abitibi-Témiscamingue.

\section{Quelques défis à relever}

Il va sans dire que certains défis devront être relevés. C'est pourquoi l'embauche d'une coordonnatrice permettra de régler quelques problématiques potentielles concernant les familles, d'une part, la commercialisation et la promotion du produit, d'autre part. Bien que le mouvement initial motive grandement les parties prenantes, certaines mesures, qui exigent patience et persévérance, doivent être envisagées pour maintenir la motivation : roder le produit, prendre le temps de faire connaître la formule, perfectionner le savoir-faire, recevoir des visiteurs de façon sporadique pour commencer..

Le recrutement des familles doit également se faire de façon continue, tant pour se préparer à recevoir de plus grands groupes que pour assurer une disponibilité constante d'hébergement à l'année. 
Les activités inscrites au programme doivent être revues, évaluées et continuellement peaufinées afin de conserver l'aspect unique de la rencontre entre les visiteurs, la région et ses villageois. La recherche et le développement de nouvelles activités tant estivales qu'hivernales, ainsi que la formation des artistes, des artisans et des spécialistes en vue d'améliorer leur prestation sont d'une importance capitale à la viabilité du projet.

Il faut en outre mobiliser des ressources financières et humaines qui seront affectées au développement d'un plan stratégique de marketing et de communication. La représentation auprès des clientèles ciblées, le réseautage avec les intermédiaires de vente, la sollicitation auprès de groupes et le démarchage continu exigent du temps et des suivis constants pour que le projet produise des résultats probants. La constitution d'un fonds de roulement devient alors impérative.

\section{Les impacts du projet}

I| est déjà possible de mesurer certains impacts à la suite de l'implantation du projet : un emploi à plein temps a été créé et un autre le sera prochainement, tout comme un poste à demi-temps, sans compter les revenus d'appoint des producteurs, des artistes, des artisans et des spécialistes affectés au programme d'activités ; on entrevoit également la création et le maintien de plusieurs emplois indirects:

Au chapitre des ventes, le plan d'affaires est basé sur une capacité maximale actuelle d'environ 17472 nuitées avec 42 familles participantes (48 chambres) et vise 65 familles (74 chambres), pour une capacité prévue de près de 27000 nuitées dans les trois prochaines années, prévoyant $50 \%$ d'augmentation pour les deuxième et troisième années.

L'augmentation de la capacité d'accueil pour les quatre municipalités et l'élargissement de l'activité touristique sur quatre saisons permettent de diversifier non seulement l'offre et la notoriété touristiques, mais également l'économie de la MRC d'Antoine-Labelle.

\section{Les conditions propices au succès}

Le projet est largement appuyé par le milieu. Les quatre municipalités investissent non seulement en participant au financement du projet, mais en siégeant au conseil d'administration et en prêtant des employés et de l'équipement. De plus, le gouvernement du Québec, dans sa Politique nationale de la ruralité, a investi une somme importante qui a permis de bonifier l'étude de marché et la promotion du village d'accueil. Une subvention du CLD, issue du fonds d'économie sociale, a permis l'embauche d'une coordonnatrice ; le CLD a également consenti à financer le salaire et les frais encourus pour les deux employées responsables du projet. Par ailleurs, ce projet a remporté les honneurs du concours d'entreprenariat de la MRC d'Antoine-Labelle en 2006. II a été choisi comme "Bon Coup de l'année» et s'est classé parmi les cinq meilleurs projets de l'Association des CLD du Québec.

La crédibilité des leaders dans chaque municipalité, le respect mutuel des intervenants municipaux entre eux, la transparence, la vision communautaire ainsi que la gestion participative sont autant de conditions favorables au succès du projet. Le principe de l'intention (Pine et Gilmore, 1999: 202) doit donc faire partie des stratégies mises en place pour conserver cet esprit d'authenticité recherché autant par le visité que par le visiteur.

Certains incitatifs ont également été intégrés au projet. En premier lieu, les familles et les chargés d'activités seront rémunérés de façon appropriée. Ils recevront un bulletin d'information mensuel et auront accès à des ateliers de perfectionnement continu (accueil, histoire, cuisine, partenariats avec les producteurs locaux, meilleure connaissance de leur milieu et de leur potentiel de développement futur). Ils participeront à des activités d'échanges et seront secondés dans l'amélioration de leur prestation. Enfin, les responsables du projet sont à mettre sur pied une carte d'achats privilège auprès des producteurs locaux, afin d'encourager ces derniers et donner des rabais aux familles hôtes. II va sans dire que des stratégies de commercialisation et des outils promotionnels, dont un site web, [www.villagedaccueil.com], ont été élaborés dans le but de rejoindre facilement les clientèles ciblées.

\section{Conclusion}

Le projet a connu un tel succès auprès de la population que certains terrains stratégiques ont été cédés pour assurer un décor ou un lieu propice aux activités programmées. Une maison de bois rond qui servira à des ateliers d'apprentissage est présentement en construction. Par ailleurs, une entreprise de fabrication de maisons pièce sur pièce de la municipalité de Lac-du-Cerf construira une maison (qui sera financée par un grand tirage provincial) au bord d'un lac, sur un terrain qui sera cédé par la MRC d'Antoine-Labelle en guise de contribution au financement du projet.

Revenir à une échelle humaine, utiliser les expertises locales, faire briller les talents de chacun, accueillir dans le meilleur sens du terme, voilà en quelques mots l'esprit de ce projet communautaire. Le goût et le pouvoir d'influencer leur développement et leur futur donne aux communautés un élan de solidarité rurale par le biais de personnes qui ont foi dans le projet et qui font preuve d'imagination et de dévouement pour l'amener à terme ${ }^{5}$.

Henry Mintzberg, célèbre universitaire canadien en sciences de la gestion, n'inventait-il pas récemment le mot communautéship «pour décrire les processus collectifs qui mènent à des réalisations remarquables » : "En reconnaissant le communautéship, le leadership ne disparaît pas. [...] Nous sommes la solution aux problèmes de ce monde. Nous tous, si nous travaillons ensemble » (Tremblay, 2007).

Marie-Andrée Delisle dirige sa propre firme de services conseils en développement touristique. Elle travaille étroitement avec le CLD d'Antoine-Labelle sur ce projet de village d'accueil depuis ses débuts.

\section{Notes}

1 Règlement sur les établissements d'hébergement touristique, c. E-14.2, r.01, art. 7 .

2 La Corporation de l'industrie touristique du Québec (CITQ) a été mandatée pour assurer le développement et l'implantation de la classification des établissements d'hébergement touristique du Québec. 
3 Conformément aux dispositions de la Loi sur les établissements d'hébergement touristique, L.R.Q., C. E-14.2, art. 6-10, et du Règlement sur les établissements d'hébergement touristique (op. cit.), art. 7 : [http:// www.bonjourquebec.com/mto/program mes/hebergement/index.html], (consulté le 23 juin 2007).

4 Ces municipalités avaient déjà collaboré à d'autres projets, mais la motivation et les opportunités de travailler ensemble s'étaient perdues au fil du temps.

5 Dans son livre sur l'écotourisme et la gouvernance participative, Marie Lequin (2001: 25) considère ce type de projet comme étant de l'écotourisme, en soulignant que "l'écotourisme, comme stratégie de développement régional, est en partie fondé sur la participation de la communauté à la gestion de son patrimoine environnemental et culturel et sur le rôle décisif qu'elle joue ainsi dans le développement et la mise en marché du produit. Un écotourisme durable est celui qui permet de satisfaire les besoins des touristes actuels et des communautés d'accueil tout en préservant et augmentant le potentiel d'avenir.
En d'autres termes, l'écotourisme durable repose sur le processus d'interaction avec l'environnement et les échanges culturels avec les communautés d'accueil. "

Dans le même ordre d'idées, l'émission estivale télévisée La Petite Séduction (diffusée à Radio-Canada) a permis de mieux comprendre la force d'un projet communautaire lorsqu'on s'y met. L'idée de l'émission est d'associer un ou une artiste à un village et de faire de ce dernier un havre pouvant correspondre aux goûts et aux intérêts de l'invité de la semaine. Les villageois sont donc invités à imaginer des scénarios pour charmer l'invité tout en faisant valoir les talents de la communauté. L'émission de 30 minutes, qui vient de terminer sa deuxième saison et en prépare une troisième, fait visiter 19 villages chaque été. L'exercice n'est pas toujours entièrement réussi, mais la synergie de ce mouvement collectif est toujours présente et montre combien la solidarité des gens peut engendrer une force incroyable lorsqu'ils se concentrent sur un but commun et misent sur leurs aspects distinctifs et leur complémentarité plutôt que sur leurs différences.

\section{Bibliographie}

Delisle, Marie-Andrée, et Louis Jolin (2007), Un autre tourisme est-il possible?, Québec, Presses de l'Université du Québec.

Lacasse, Josée, Alain Morin, Angèle Nantel, et Solange Nantel (1998), Kiamika comme une rivière..., Kiamika, Comité des fêtes du centenaire de Kiamika.

Le Groupe DBSF (2007), Plan stratégique de développement et de marketing 2007-2010, Rapport final pour le compte de l'Association des villages d'accueil du Québec.

Lequin, Marie (2001), Écotourisme et gouvernance participative, Presses de l'Université du Québec.

Pine II, Joseph B., et James H. Gilmore (1999), The Experience Economy, Boston, Harvard Business School Press.

Tremblay, Jacynthe (2007), « Entretien avec Henry Mintzberg : De l'obsession du leadership au communautéship ", La Presse, 14 avril, Cahier Affaires, p. 5.

Village d'accueil des Hautes-Laurentides, [www. villagedaccueil.com/], (consulté le 20 juillet 2007).

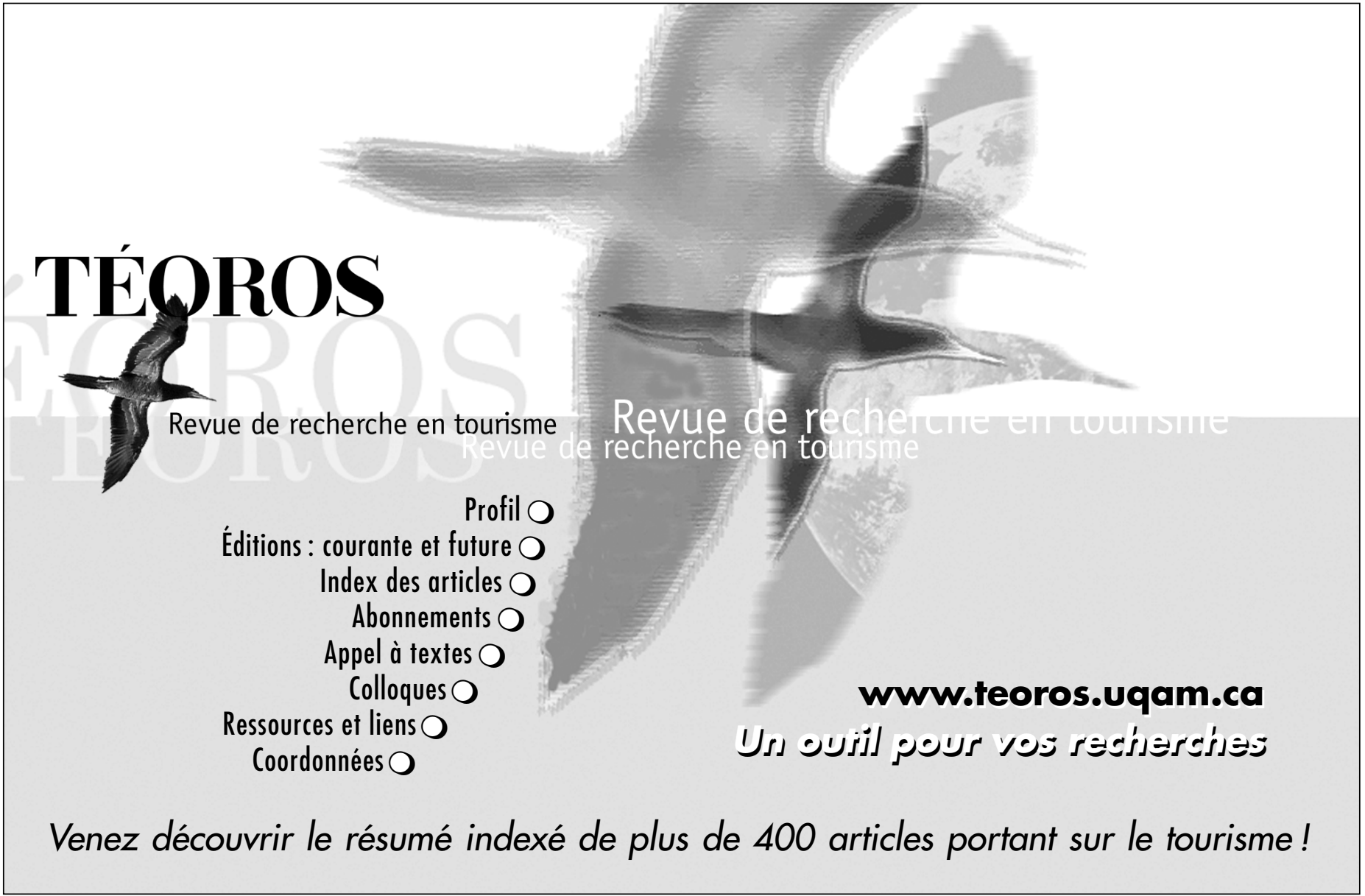

